\title{
Effect of vitamin D supplementation on selected inflammatory biomarkers in older adults: a secondary analysis of data from a randomised, placebo-controlled trial
}

\author{
Mary Waterhouse ${ }^{1,2} \dagger$, Bich $\operatorname{Tran}^{3} \dagger$, Peter R. Ebeling ${ }^{2,4}$, Dallas R. English ${ }^{5,6}$, Robyn M. Lucas ${ }^{7,8}$, \\ Alison J. Venn ${ }^{9}$, Penelope M. Webb ${ }^{1}$, David C. Whiteman ${ }^{1,2}$ and Rachel E. Neale ${ }^{1,2_{*}}$ \\ ${ }^{1}$ Population Health Division, QIMR Berghofer Medical Research Institute, Herston, Queensland 4006, Australia \\ ${ }^{2}$ Centre for Research Excellence in Sun and Health, Kelvin Grove, Queensland 4059, Australia \\ ${ }^{3}$ Centre for Big Data Research in Health, Faculty of Medicine, University of New South Wales, Sydney, NSW 2052, Australia \\ ${ }^{4}$ Department of Medicine, Monash University, Victoria 3800, Australia \\ ${ }^{5}$ Centre for Epidemiology and Biostatistics, University of Melbourne, Melbourne, Victoria 3010, Australia \\ ${ }^{6}$ Cancer Epidemiology Centre, Cancer Council Victoria, Melbourne, Victoria 3004, Australia \\ ${ }^{7}$ National Centre for Epidemiology and Population Health, Australian National University, Canberra, Australian Capital \\ Territory 0200, Australia \\ ${ }^{8}$ Telethon Kids Institute, University of Western Australia, Perth, Western Australia 6009, Australia \\ ${ }^{9}$ Menzies Research Institute Tasmania, University of Tasmania, Hobart, Tasmania 7000, Australia
}

(Submitted 24 March 2015 - Final revision received 10 May 2015 - Accepted 2 June 2015 - First published online 24 July 2015)

\section{Abstract}

Observational studies have suggested that 25-hydroxyvitamin D (25(OH)D) levels are associated with inflammatory markers. Most trials reporting significant associations between vitamin $\mathrm{D}$ intake and inflammatory markers used specific patient groups. Thus, we aimed to determine the effect of supplementary vitamin D using secondary data from a population-based, randomised, placebo-controlled, doubleblind trial (Pilot D-Health trial 2010/0423). Participants were 60- to 84-year-old residents of one of the four eastern states of Australia. They were randomly selected from the electoral roll and were randomised to one of three trial arms: placebo $(n 214), 750 \mu \mathrm{g}(n 215)$ or $1500 \mu \mathrm{g}(n$ 215) vitamin $D_{3}$, each taken once per month for 12 months. Post-intervention blood samples for the analysis of C-reactive protein (CRP), IL-6, IL-10, leptin and adiponectin levels were available for 613 participants. Associations between intervention group and biomarker levels were evaluated using quantile regression. There were no statistically significant differences in distributions of CRP, leptin, adiponectin, leptin: adiponectin ratio or IL-10 levels between the placebo group and either supplemented group. The 75 th percentile IL- 6 level was $2 \cdot 8 \mathrm{pg} / \mathrm{ml}$ higher $(95 \% \mathrm{CI} 0 \cdot 4,5 \cdot 8 \mathrm{pg} / \mathrm{ml})$ in the $1500 \mu \mathrm{g}$ group than in the placebo group (75th percentiles:11.0 $v .8 \cdot 2 \mathrm{pg} / \mathrm{ml})$, with a somewhat smaller, non-significant difference in 75th percentiles between the $750 \mu \mathrm{g}$ and placebo groups. Despite large differences in serum $25(\mathrm{OH}) \mathrm{D}$ levels between the three groups after 12 months of supplementation, we found little evidence of an effect of vitamin D supplementation on cytokine or adipokine levels, with the possible exception of IL-6.

Key words: Vitamin D: Supplementation: Cytokines: Adipokines

Chronic inflammation underlies many pathological processes including several types of cancer ${ }^{(1)}, \mathrm{CVD}^{(2)}$ and autoimmune disease ${ }^{(3)}$. Animal and in vitro studies have identified a number of mechanisms by which the most biologically active form of vitamin $\mathrm{D}, 1,25$-dihydroxyvitamin $\mathrm{D}\left(1,25(\mathrm{OH})_{2} \mathrm{D}\right)$, may reduce inflammation $^{(4)}$. For example, treatment with $1,25(\mathrm{OH})_{2} \mathrm{D}$ suppresses the NF- $\kappa \mathrm{B}$ pathway ${ }^{(5)}$, thereby reducing downstream expression of pro-inflammatory cytokines. By acting as a ligand-activated transcription factor, the vitamin $\mathrm{D}$ receptor
(VDR) can also directly regulate cytokine gene transcription ${ }^{(6)}$. In addition, the VDR is expressed in adipocytes that are responsible for the production of the adipokines adiponectin and leptin. These have anti- and pro-inflammatory properties, respectively, but investigations of the influence of $1,25(\mathrm{OH})_{2} \mathrm{D}$ on their secretion are scarce ${ }^{(7,8)}$.

Serum concentrations of the intermediate product 25-hydroxyvitamin D (25(OH)D) are used to measure a person's vitamin D status. Many observational studies suggest

Abbreviations: 25(OH)D, 25-hydroxyvitamin D; CRP, C-reactive protein; LAR, leptin:adiponectin ratio; RCT, randomised controlled trial; QR, quantile regression.

* Corresponding author: Dr R. E. Neale, fax +61 73845 3502, email rachel.neale@qimrberghofer.edu.au

$\dagger$ These authors contributed equally to this work. 
that $25(\mathrm{OH}) \mathrm{D}$ concentration is inversely associated with pro-inflammatory markers and positively associated with anti-inflammatory markers $^{(9-14)}$. For example, studies have reported an inverse relationship between serum 25(OH)D levels and circulating concentrations of C-reactive protein $(\mathrm{CRP})^{(\mathrm{O}, 10)}$, IL- $6^{(9)}$ and leptin ${ }^{(11)}$, and a positive association with adiponectin levels ${ }^{(12-14)}$. Other studies suggest a less straightforward relationship. Both pro- and anti-inflammatory cytokines levels were found to be down-regulated in summer in association with increased serum $25(\mathrm{OH}) \mathrm{D}$ levels ${ }^{(15)}$. In contrast, an observational analysis of data from a trial suggested that supplementation with vitamin $\mathrm{D}$ increased the levels of both pro- and anti-inflammatory cytokines in people with initially low serum 25(OH)D levels ${ }^{(16)}$.

In some trials, supplementation with vitamin $\mathrm{D}$ has been associated with significant reductions in circulating concentrations of $\mathrm{CRP}^{(17,18)}$ and IL-6 $6^{(19,20)}$, and significant increases in IL-10 ${ }^{(21)}$ and adiponectin ${ }^{(22)}$. However, most trials with significant results were carried out in patients with a specific disease condition rather than in 'healthy' participants. Results from trials that used people without apparent disease were largely null, although these used relatively small sample sizes $(n \text { 23-307 })^{(23-29)}$. A pooled analysis of data from four trials, none of which was populationbased, found no significant change in CRP following high-dose supplementation with vitamin $\mathrm{D}^{(30)}$.

Many people without a specific disease may still have chronic, low-grade inflammation, especially if exposed to toxic chemicals (e.g. tobacco smoke), or if overweight or obese ${ }^{(31,32)}$. Given the possible consequences for the prevention of cancer and CVD, it is worth investigating whether vitamin D supplementation can dampen subclinical inflammation in apparently healthy people. We used secondary data from our randomised controlled trial (RCT) of vitamin D supplementation in a population-based sample of older Australians, which demonstrated large effects of supplementation on serum $25(\mathrm{OH}) \mathrm{D}^{(33)}$, to assess the effect of supplementary vitamin D on circulating inflammatory markers (CRP, leptin, adiponectin, leptin:adiponectin ratio (LAR), IL-6 and IL-10). To the best of our knowledge, this is one of the largest and, hence, most highly powered studies of the possible effect of vitamin $\mathrm{D}$ on inflammation, with relevance extending beyond highly specific patient groups.

\section{Methods}

\section{Study population and design}

The methods and protocols of the study have been described ${ }^{(33)}$. Briefly, the Pilot D-Health trial 2010/0423 was a populationbased, randomised, placebo-controlled, double-blind chemoprevention trial of vitamin $\mathrm{D}_{3}$ in older adults. This study was conducted according to the guidelines laid down in the 1964 Declaration of Helsinki, and all procedures involving human subjects were approved by The Human Research Ethics Committee at the QIMR Berghofer Medical Research Institute. Written informed consent was obtained from all participants. Participants consented to blood samples being stored indefinitely and for them to be used in studies related to vitamin D. The QIMR Berghofer Medical Research Institute Human Research Ethics Committee approved the use of samples for this study.
We recruited 644 people aged $60-84$ years who were residents of one of the four eastern states of Australia, using the Australian Electoral Roll as the sampling frame. Participants were sampled in strata of 5-year age bands, sex, state and location of residence (capital city and elsewhere). We excluded anybody who was taking $\geq 10 \mu \mathrm{g}$ of vitamin $\mathrm{D} / \mathrm{d}$ or who had a history of kidney stones, hyperparathyroidism, osteomalacia, osteoporosis or sarcoidosis. Recruitment took place between October 2010 and March 2011, and $10 \%$ of people invited were randomised. Participants were randomly allocated to one of three trial arms: placebo ( $n$ 214), $750 \mu \mathrm{g}(n$ 215), or $1500 \mu \mathrm{g}$ ( $n$ 215) vitamin $\mathrm{D}_{3}$ (cholecalciferol), each taken once per month for 12 months. Participants, study investigators and staff were unaware of the treatment allocation until analysis of the primary outcome (change in serum $25(\mathrm{OH}) \mathrm{D}$ level) was complete. Approximately $95 \%$ of participants completed the study and $96 \%$ reported taking at least ten of their twelve study tablets.

\section{Blood samples and laboratory analyses}

Non-fasting blood samples were collected in serum separator tubes at study entry and after the intervention period and transported cold by overnight courier to the Queensland University of Technology (Brisbane, Australia). Participants were requested to provide a post-intervention blood sample within 2 weeks of the last tablet taken. Samples were centrifuged at $4^{\circ} \mathrm{C}$ for $15 \mathrm{~min}$ at $2000 \mathrm{~g}$, and the serum was stored at $-80^{\circ} \mathrm{C}$.

Pre- and post-intervention serum $25(\mathrm{OH}) \mathrm{D}$ concentrations were measured in a single batch using a commercial chemiluminescent immunoassay (LIAISON ${ }^{\circledR}$ 25(OH)D Vitamin D TOTAL Assay; DiaSorin, Inc.). Intra- and inter-assay variabilities were 3-6 and 6-9\%, respectively. The laboratory undertaking the testing was certified by the international Vitamin D External Quality Assessment Scheme.

We measured non-fasting serum levels of five inflammatory markers in post-intervention blood samples: IL-6, IL-10, leptin, adiponectin and CRP. Levels of IL-6, IL-10, leptin and adiponectin were measured using commercial sandwich ELISA kits (Merck Millipore) using a Multiskan Primary EIA V. 2.3 device with Labsystems Genesis V3.03 software, and levels of CRP were measured using the high-sensitivity CardioPhase hsCRP assay on the $\mathrm{BN}^{\mathrm{TM}}$ II system (Siemens Healthcare Diagnostics). The inter- and intra-assay CV were $<10$ and $<15 \%$ for all biomarkers. The minimum detectable concentrations for IL-6, IL-10, leptin, adiponectin and CRP were $1.6 \mathrm{pg} / \mathrm{ml}, 2 \mathrm{pg} /$ $\mathrm{ml}, \quad 0.78 \mathrm{ng} / \mathrm{ml}, 0.1 \mu \mathrm{g} / \mathrm{ml}$ and $0.175 \mathrm{mg} / \mathrm{l}$, respectively. All assays for inflammatory markers were performed by Sullivan Nicolaides Pathology (Indooroopilly, Australia) according to the manufacturers' instructions.

\section{Questionnaires}

Within 2 weeks of having blood drawn at study entry and exit, participants were asked to complete questionnaires in which they reported information about their height, weight, skin phenotype, smoking and alcohol consumption, history of cancer, diabetes or CVD, physical activity, time outdoors, overall health and dietary intake of vitamin D. 


\section{Statistical analysis}

We excluded participants for whom no blood sample was available for the analysis of inflammatory markers. Cytokine levels below the minimum detectable concentration were assigned a value equal to the minimum detectable concentration for that biomarker. Adipokine levels outside the appropriate range of the assay were assigned the lower or upper bound as appropriate. The $\mathrm{LAR}^{(34)}$ was calculated by dividing the leptin level $(\mathrm{ng} / \mathrm{ml})$ by the adiponectin level $(\mu \mathrm{g} / \mathrm{ml})$.

Comparisons of post-intervention levels of serum 25(OH)D were made between intervention groups using two-sample $t$ tests. As biomarker distributions were right-skewed, we present their descriptive statistics as median (interquartile range (IQR)).

We used quantile regression $(\mathrm{QR})$ to evaluate the association between intervention group and each biomarker level. QR is used to model a specified percentile of a response variable, conditional on one or more predictors. It is therefore similar to linear regression, which models the conditional mean of the response variable. The distribution of some biomarkers remained rightskewed following a log transformation. QR makes no assumptions regarding the distribution of the regression residuals and therefore offered an advantage over linear regression. Further, QR was preferable to Wilcoxon's rank sum test because it permitted the inclusion of interaction terms.

We compared median levels of CRP, leptin, adiponectin and LAR, and 75th percentile levels of IL-6 and IL-10. These were meaningful comparisons given the shapes of biomarker distributions. In particular, as IL-6 and IL-10 levels were very right-skewed, we were interested in whether vitamin D supplementation might have a dampening effect on more 'extreme' levels of these biomarkers.

Intervention group was treated as a categorical predictor in $\mathrm{QR}$ models, with the placebo group used as the reference level. For $\mathrm{CRP}$, leptin, adiponectin and LAR, QR coefficients represent the estimated difference in medians between an actively supplemented group (either the 750 or $1500 \mu$ group) and the placebo group. For IL- 6 and IL-10, the coefficients represent the estimated difference in 75th percentiles. $95 \%$ confidence intervals for each coefficient are interpreted in the usual way; a CI that does not contain 0 indicates that the percentile differs significantly between the placebo and supplemented groups. Wald's statistic was used to assess the overall significance of the intervention group. To test for linear trends, we fitted models with intervention group treated as a continuous variable, with the values 0,1 and 2 corresponding to the placebo, 750 and $1500 \mu \mathrm{g}$ groups, respectively.

To test whether the effect of supplementation was modified by sex, age $\left(<70, \geq 70\right.$ years), baseline BMI $\left(<25, \geq 25 \mathrm{~kg} / \mathrm{m}^{2}\right)$, baseline serum $25(\mathrm{OH}) \mathrm{D}$ level $(<30, \geq 30 \mathrm{nmol} / \mathrm{l})$ or time between last tablet and collection of post-intervention blood sample $(\leq 7,>7 d)$, we included interaction terms for intervention group by each predictor. The cutoff point of $30 \mathrm{nmol} / \mathrm{l}$ was selected based on the Institute of Medicine's 2011 report $^{(35)}$. Analyses were repeated within strata of sex, age, baseline BMI, baseline serum $25(\mathrm{OH}) \mathrm{D}$ level and timing of blood collection.

Analyses were performed in SAS version 9.2 (SAS Institute, Inc.). All $P$ values are two-sided, and we used a statistical significance level of $P<0 \cdot 05$.
Sample size. The trial was designed to have adequate power to assess changes in serum $25(\mathrm{OH}) \mathrm{D}$ levels ${ }^{(33)}$. The final number enrolled was greater than planned because of an increase in response rate in later waves of recruitment.

Given strong recommendations against their use ${ }^{(36)}$, post hoc power calculations have not been performed for this secondary analysis of inflammatory markers. This is, however, one of the largest randomised placebo-controlled trials conducted to date that investigates the effect of vitamin D supplementation on inflammatory markers.

\section{Results}

\section{Characteristics of participants}

We randomised 644 participants, 615 of whom completed the study. Post-intervention blood samples for the analysis of inflammatory markers were available for 613 participants. The median time between the last tablet taken and collection of the post-intervention blood sample was 7 (IQR 6-11) d and, within each treatment group, $93 \%$ of people provided the sample within 2 weeks of taking the final tablet. The average age of participants included in this analysis was 72 (SD 7) years, and $46 \%$ were women. Baseline characteristics are presented in Table 1. Baseline serum 25(OH)D levels were similar for the three groups (Table 1), whereas average post-intervention serum $25(\mathrm{OH}) \mathrm{D}$ levels were highest in the $1500 \mu \mathrm{g}$ group (78 (sD 20) $\mathrm{nmol} / \mathrm{l})$, intermediate in the $750 \mu \mathrm{g}$ group (64 (sD 17) $\mathrm{nmol} / \mathrm{l}$ ) and lowest (unchanged) in the placebo group (42 (SD 15) nmol/l) ${ }^{(33)} ; \quad P$ values for all pairwise comparisons were $<0 \cdot 0001$. The percentage of participants with serum 25(OH)D levels $>75 \mathrm{nmol} / 1$ also significantly differed between groups (1500 $\mu \mathrm{g}$ group, $54 \%$; $750 \mu \mathrm{g}$ group, $24 \%$; placebo group, $1 \%$ ).

\section{Analysis of inflammatory markers}

Each of CRP, leptin, adiponectin and LAR had one missing observation, and data were missing for IL-6 and IL-10 in four and twelve cases, respectively. The percentages of observations with a biomarker level below the limit of detection were $1 \%$ (CRP), $32 \%$ (IL-6), $58 \%$ (IL-10), 3\% (leptin) and 2\% (adiponectin); the percentages did not differ between treatment groups. The 75 th percentile IL-6 level was $2.8 \mathrm{pg} / \mathrm{ml}$ higher $(95 \%$ CI $0.4,5 \cdot 8 \mathrm{pg} / \mathrm{ml})$ in the $1500 \mu \mathrm{g}$ group than in the placebo group (75th percentiles: 11.0 v. $8.2 \mathrm{pg} / \mathrm{ml}$ ) (Table 2). There was a somewhat smaller and non-significant difference (in 75th percentile IL-6 levels) between the $750 \mu \mathrm{g}$ and placebo groups (Table 2). There were no statistically significant differences in median levels of CRP, leptin, adiponectin or LAR, or in 75th percentile levels of IL-10 levels, between the placebo group and either the 750 or the $1500 \mu$ group (Table 2).

There was a significant interaction between the intervention group and age with respect to the association between supplementation and median levels of leptin and LAR ( $P$ interaction $=0.03$ and 0.02 , respectively) (online Supplementary Table 51 ). For people aged $<70$ years, the median leptin and LAR levels were higher in the $750 \mu \mathrm{g}$ group and 
Table 1. Baseline characteristics of participants by treatment group

(Mean values with their standard deviations; percentages)

\begin{tabular}{|c|c|c|c|c|c|c|}
\hline \multirow[b]{2}{*}{ Variable } & \multicolumn{2}{|c|}{ Placebo ( $n$ 205) } & \multicolumn{2}{|c|}{$750 \mu \mathrm{g}(n 203)$} & \multicolumn{2}{|c|}{$1500 \mu \mathrm{g}(n 205)$} \\
\hline & Mean & SD & Mean & SD & Mean & SD \\
\hline Age (years) & 71.5 & $6 \cdot 8$ & 71.6 & $7 \cdot 0$ & 71.5 & $6 \cdot 8$ \\
\hline BMl $\left(\mathrm{kg} / \mathrm{m}^{2}\right)^{\star}$ & 27.5 & 4.7 & 27.3 & 4.6 & $27 \cdot 2$ & 4.1 \\
\hline Baseline serum $25(\mathrm{OH}) \mathrm{D}(\mathrm{nmol} / \mathrm{l}) \dagger$ & $42 \cdot 0$ & 13.5 & 41.6 & $12 \cdot 7$ & 41.9 & $14 \cdot 3$ \\
\hline Physical activity (METs/week) & $45 \cdot 7$ & $45 \cdot 2$ & $46 \cdot 6$ & $49 \cdot 1$ & $46 \cdot 0$ & $52 \cdot 7$ \\
\hline Time outdoors (h/week) & $14 \cdot 0$ & $12 \cdot 0$ & $15 \cdot 0$ & $12 \cdot 7$ & $14 \cdot 7$ & $12 \cdot 3$ \\
\hline Vitamin D intake $(\mu \mathrm{g} / \mathrm{d})$ & $3 \cdot 1$ & $3 \cdot 6$ & 3.3 & $4 \cdot 8$ & $3 \cdot 4$ & 4.3 \\
\hline \multicolumn{7}{|l|}{$\operatorname{Sex}(\%)$} \\
\hline Men & \multicolumn{2}{|c|}{54.6} & \multicolumn{2}{|c|}{54.7} & \multicolumn{2}{|c|}{$51 \cdot 7$} \\
\hline Women & \multicolumn{2}{|c|}{$45 \cdot 4$} & \multicolumn{2}{|c|}{$45 \cdot 3$} & \multicolumn{2}{|c|}{$48 \cdot 3$} \\
\hline \multicolumn{7}{|l|}{ Alcohol consumption (drinks/week) (\%) } \\
\hline$\leq 1$ & \multicolumn{2}{|c|}{$45 \cdot 9$} & \multicolumn{2}{|c|}{$42 \cdot 4$} & \multicolumn{2}{|c|}{$43 \cdot 9$} \\
\hline $2-6$ & \multicolumn{2}{|c|}{$21 \cdot 0$} & \multicolumn{2}{|c|}{$22 \cdot 7$} & \multicolumn{2}{|c|}{$27 \cdot 3$} \\
\hline $7-20$ & \multicolumn{2}{|c|}{$25 \cdot 4$} & \multicolumn{2}{|c|}{$28 \cdot 6$} & \multicolumn{2}{|c|}{$23 \cdot 4$} \\
\hline$\geq 21$ & \multicolumn{2}{|c|}{$7 \cdot 8$} & \multicolumn{2}{|c|}{$6 \cdot 4$} & \multicolumn{2}{|c|}{$5 \cdot 4$} \\
\hline \multirow{2}{*}{\multicolumn{7}{|c|}{ Smoking status (\%) }} \\
\hline Never smoker & \multicolumn{2}{|c|}{$57 \cdot 1$} & \multicolumn{2}{|c|}{$58 \cdot 1$} & & $60 \cdot 0$ \\
\hline Ex-smoker & \multicolumn{2}{|c|}{38.5} & \multicolumn{2}{|c|}{35.5} & \multirow{2}{*}{\multicolumn{2}{|c|}{$36 \cdot 1$}} \\
\hline Current smoker & \multicolumn{2}{|c|}{4.4} & \multicolumn{2}{|c|}{6.4} & & 3.9 \\
\hline Self-reported health status (\%) & & & & & & \\
\hline Good & & & & & & \\
\hline Fair/poor & & & & & & \\
\hline
\end{tabular}

25(OH)D, 25-hydroxyvitamin D; MET, metabolic equivalent task.

* Missing data for one participant in the placebo group.

$\dagger$ Missing data for one participant in the $1500 \mu \mathrm{g}$ group.

Table 2. Comparison of inflammatory markers following 12 months of monthly supplementation with placebo, or 750 or $1500 \mu g$ vitamin $D_{3}$ : quantile regression* (Medians, interquartile ranges, $b$ values and $95 \%$ confidence intervals)

\begin{tabular}{|c|c|c|c|c|c|c|c|c|c|c|c|c|}
\hline \multirow[b]{2}{*}{ Variable } & \multicolumn{2}{|c|}{ Placebo ( $n$ 205) } & \multicolumn{4}{|c|}{$750 \mu \mathrm{g}$ vitamin $\mathrm{D}_{3}(n$ 203) } & \multicolumn{4}{|c|}{$1500 \mu \mathrm{g}$ vitamin $D_{3}(n$ 205) } & \multirow[b]{2}{*}{$P \dagger$} & \multirow[b]{2}{*}{$P$ trend $\neq$} \\
\hline & Median & IQR & Median & IQR & $b$ & $95 \% \mathrm{Cl}$ & Median & IQR & $b$ & $95 \% \mathrm{Cl}$ & & \\
\hline CRP & 1.3 & -3.4 & 1.5 & $0.8-3 \cdot 2$ & 0.1 & -0.3 & 1.4 & $0.8-3.4$ & 0.1 & $-0.3,0.6$ & 0.80 & 0.58 \\
\hline Leptin $(\mathrm{ng} / \mathrm{ml}) \S$ & 8.2 & $3 \cdot 1-17 \cdot 7$ & $8 \cdot 1$ & $2 \cdot 9-16 \cdot 6$ & -0.1 & $-2 \cdot 8,2 \cdot 1$ & 8.7 & $4 \cdot 0-15 \cdot 4$ & 0.5 & $-2 \cdot 4,2 \cdot 7$ & 0.89 & 0.76 \\
\hline Adiponectin $(\mu \mathrm{g} / \mathrm{ml}) \S$ & $13 \cdot 6$ & $8 \cdot 0-20 \cdot 7$ & $12 \cdot 8$ & $7 \cdot 9-19.5$ & -0.9 & $-3 \cdot 0,1 \cdot 4$ & $12 \cdot 6$ & $7 \cdot 8-19 \cdot 6$ & $-1 \cdot 0$ & $-2 \cdot 7,1 \cdot 0$ & 0.63 & 0.37 \\
\hline LAR§ & 0.6 & $0.3-1.5$ & 0.6 & $0.3-1.5$ & 0.1 & $-0 \cdot 2,0 \cdot 2$ & 0.7 & $0.3-1.4$ & 0.1 & $-0.1,0.3$ & 0.64 & 0.38 \\
\hline IL-6 (pg/ml)\| & $4 \cdot 0$ & $1 \cdot 6-8.2$ & 4.5 & $1.6-9.6$ & 1.4 & $-1 \cdot 4,4.5$ & 4.6 & $1.6-11.0$ & $2 \cdot 8$ & $0.4,5.8$ & 0.24 & 0.10 \\
\hline $\mathrm{IL}-10(\mathrm{pg} / \mathrm{ml}) \mathbb{|}$ & $2 \cdot 0$ & $2 \cdot 0-4 \cdot 2$ & $2 \cdot 0$ & $2 \cdot 0-5 \cdot 3$ & $1 \cdot 0$ & $-1 \cdot 2,4 \cdot 2$ & $2 \cdot 0$ & $2 \cdot 0-3 \cdot 8$ & -0.3 & $-2 \cdot 3,2 \cdot 2$ & 0.55 & 0.68 \\
\hline
\end{tabular}

IQR, interquartile range; CRP, C-reactive protein; LAR, leptin:adiponectin ratio.

${ }^{*}$ For CRP, leptin, adiponectin and LAR, quantile regression coefficients (denoted $b$ ) represent the estimated difference in medians between the actively supplemented group (750 or $1500 \mu \mathrm{g})$ and the placebo group. For IL- 6 and IL-10, $b$ represents the estimated difference in 75 th percentiles.

t $P$ value from the Wald test for intervention group treated as a categorical predictor (with $2 \mathrm{df}$ ), with the placebo group used as the reference level.

$\ddagger P$ trend from the Wald test for intervention group treated as a continuous variable (1 df) with values 0,1 and 2 corresponding to the placebo, 750 and $1500 \mu \mathrm{g}$ groups, respectively.

$\S$ Missing data for one participant in the placebo group.

II Missing data for three participants in the placebo group and one participant in the $750 \mu \mathrm{g}$ group.

II Missing data for four, three and five participants in the placebo, 750 and $1500 \mu \mathrm{g}$ groups, respectively.

lower in the $1500 \mu \mathrm{g}$ group compared with the placebo group. The opposite pattern was observed for those aged at least 70 years. There were no other significant interactions.

\section{Discussion}

Despite large differences in serum 25(OH)D levels between the three groups after 12 months of vitamin D supplementation, we found little evidence of an effect of supplementation on levels of cytokines and adipokines, with the possible exception of IL-6. The significantly higher 75th percentile IL-6 level in the $1500 \mu \mathrm{g}$ group compared with the placebo group was in contrast to our expectations.
There have been at least ten RCT examining the effect of vitamin D supplementation on IL-6 or IL-10 concentrations. Of the RCT that included participants either without a specific health condition ${ }^{(23-26)}$ or that only included overweight or obese participants ${ }^{(27-29)}$, the results for IL-6 and IL-10 were all essentially null. In one of these, there was a suggestion of a greater change in median IL-10 levels in the supplemented group $(-70 \%)$ than in the placebo group $(-50 \%)$, but the difference was not significant after adjustment for multiple testing $^{(26)}$. Possible explanations for these null results include that the sample sizes were too small, the duration or dose of supplementation was insufficient to elicit a response or that the people studied had adequate vitamin D status at baseline. 
However, for almost all of these trials, supplementation significantly increased serum $25(\mathrm{OH}) \mathrm{D}$ levels ${ }^{(24-29)}$, and in two trials ${ }^{(25,28)}$ participants had low baseline $25(\mathrm{OH}) \mathrm{D}$ levels.

It is not clear why, in the present study, the upper quartile IL-6 level was greater for those receiving the highest dose of vitamin D supplementation than in the placebo group, and it is possible that this is a chance finding. It is also possible that vitamin D supplementation might be detrimental at higher doses. However, in addition to having pro-inflammatory effects, there is also evidence that IL-6 exhibits anti-inflammatory properties ${ }^{(37,38)}$. Further, in some in vitro studies using human cells, treatment with $1,25(\mathrm{OH})_{2} \mathrm{D}_{3}$ increased IL- 6 production by differentiated adipocytes ${ }^{(8)}$, and induced a subpopulation of IL-6-producing T cells when administered to T lymphocytes ${ }^{(39)}$.

Results from trials measuring CRP have been mixed, with no consistent evidence of any clinically relevant effect. In many RCT in which participants did not have a specific disease, CRP levels did not significantly change ${ }^{(23-25,27-29,40)}$. Vitamin D supplementation was associated with a significant reduction in circulating concentrations of CRP in healthy pregnant women $^{(17)}$ and in patients with a history of myocardial infarction $^{(18)}$, but CRP levels increased in another trial among people who were overweight or obese ${ }^{(41)}$.

With respect to the adipokines, some observational studies have reported positive associations between serum $25(\mathrm{OH}) \mathrm{D}$ level and adiponectin levels ${ }^{(12,13)}$, with one study using a Mendelian randomisation approach to demonstrate that the association was possibly causal ${ }^{(14)}$. In contrast, an inverse correlation has been observed between serum 25(OH)D levels and circulating concentrations of leptin ${ }^{(11)}$. Trial data concerning adipokines are scarce and those that have been conducted had small sample sizes ${ }^{(22,28,42)}$. To the best of our knowledge, only one trial has reported a significant increase in adiponectin levels following vitamin D supplementation ${ }^{(22)}$. The study was restricted to participants with type 2 diabetes, and the endof-study adiponectin levels reported were much lower than in our participants. We found no difference in levels of either adipokine between placebo and active supplementation groups. The significant interaction between the intervention and age with respect to leptin and LAR levels is mostly likely a chance finding, as there was no dose-response relationship in stratified analyses.

Our results were largely null. For a given biomarker, this means that either that the effect due to supplementation was too small to be of biological importance or that the sample size was insufficient to detect a small but potentially important effect. The CI provide a range of plausible values for the actual effect size.

Cytokine regulation may be dependent on the serum 25(OH)D levels achieved by supplementation. The authors of a study that showed that IL- 6 production by human monocytes was inhibited by treatment with $\geq 75 \mathrm{nmol} / \mathrm{l}$ (but not $37.5 \mathrm{nmol} / \mathrm{l}$ ) $25(\mathrm{OH}) \mathrm{D}_{3}$, concluded that maintaining a serum $25(\mathrm{OH}) \mathrm{D}$ level $\geq 75 \mathrm{nmol} / \mathrm{l}$ was necessary for optimal anti-inflammatory effects ${ }^{(43)}$. A small observational study of young adults ( $25-42$ years) also noted that levels of selected pro-inflammatory cytokines were higher in those with serum $25(\mathrm{OH}) \mathrm{D}$ levels $\leq 80 \mathrm{nmol} / \mathrm{l}$ when compared with people with higher levels ${ }^{(44)}$. Approximately one half of our participants in the $1500 \mu \mathrm{g}$ group achieved a postintervention serum $25(\mathrm{OH}) \mathrm{D}$ level $\geq 75 \mathrm{nmol} / 1$, compared with just $1 \%$ in the placebo group. Thus, for comparisons between the $1500 \mu \mathrm{g}$ group and the placebo group, it seems unlikely that our mostly null results are because of inadequate serum $25(\mathrm{OH})$ D levels following supplementation.

This study has some limitations. Diet influences systemic inflammation, and thus it was not ideal to have used non-fasting blood samples. Although this has the potential to influence our summary statistics, the randomisation eliminates any risk of biased estimates of treatment effect that might have otherwise resulted. Because we did not measure baseline levels of inflammatory markers, we could not assess baseline equivalence and could not analyse changes in levels. The lack of baseline data is extremely unlikely to have led to any bias in these results. The trial groups were extremely well balanced at baseline ${ }^{(45)}$, and it would be extraordinary if the distribution of any biomarkers differed before the intervention. Thus, any differences after the intervention are an indication of change from baseline. We used a monthly bolus dose of vitamin D, which some have argued might confer suboptimal benefits (for some endpoints) when compared with daily dosing ${ }^{(46-48)}$. However, two studies found that daily or monthly dosing did not produce significantly different serum 25(OH)D levels after 2 months ${ }^{(49)}$ or 1 year ${ }^{(50)}$ of vitamin $\mathrm{D}_{3}$ supplementation. Given the relatively long half-life of vitamin $\mathrm{D}$ and $25(\mathrm{OH}) \mathrm{D}^{(51)}$, variability in timing of post-intervention blood samples should also not be responsible for our largely null findings. Although non-differential follow-up could introduce bias, our extremely high completion rate (96\%) means that any bias would be negligible and would not have materially influenced the results. Caution should also be exercised in generalising our results, as our population was older and predominantly (95\%) Caucasian.

Our study also has strengths. It is one of the largest randomised placebo-controlled trials with measurement of inflammatory markers conducted to date, and, importantly, our participants were recruited from the general population. Consequently, our results may be more relevant to the general elderly population than those from studies focusing on people with specific diseases. The duration of our intervention and the doses administered were sufficient to elicit measurable increases in serum 25(OH)D levels, and our study had high rates of retention and adherence ( $96 \%$ of participants reported taking at least ten of the twelve study tablets).

With the possible exception of IL-6, we found little evidence of an effect of vitamin D supplementation on cytokine or adipokine levels. These results highlight the uncertainty surrounding the effectiveness of vitamin D supplementation in improving inflammatory biomarker profiles and the need to better understand the effects of vitamin D supplementation before recommending that it is routinely used in the general population.

\section{Acknowledgements}

This project was funded by the National Health and Medical Research Council (NHMRC) of Australia (grant no. 613655). P. M. W., R. E. N., A. J. V., R. M. L. and D. C. W. are supported by 
Fellowships from the National Health and Medical Research Council. The investigational product was supplied free of charge by Sanofi-Aventis Healthcare Pty Ltd trading as Sanofi Consumer Healthcare, Virginia, QLD, Australia.

M. W. performed the statistical analysis. B. T. and M. W. wrote the manuscript. R. E. N. coordinated the study, contributed to writing the manuscript and provided overall supervision. All authors contributed to study design and data interpretation and read and approved the final version of the manuscript.

P. R. E. has received prior funding from Sanofi-Aventis Healthcare. All other authors have no conflicts of interest to disclose.

\section{Supplementary material}

For supplementary material/s referred to in this article, please visit http://dx.doi.org/10.1017/S0007114515002366

\section{References}

1. Elinav E, Nowarski R, Thaiss CA, et al. (2013) Inflammationinduced cancer: crosstalk between tumours, immune cells and microorganisms. Nat Rev Cancer 13, 759-771.

2. Ross R (1999) Atherosclerosis - an inflammatory disease. $N$ Engl J Med 340, 115-126.

3. Munoz LE, Janko C, Schulze C, et al. (2010) Autoimmunity and chronic inflammation - two clearance-related steps in the etiopathogenesis of SLE. Autoimmun Rev 10, 38-42.

4. van Etten E \& Mathieu C (2005) Immunoregulation by 1,25-dihydroxyvitamin $\mathrm{D}_{3}$ : basic concepts. J Steroid Biochem Mol Biol 97, 93-101.

5. D'Ambrosio D, Cippitelli M, Cocciolo MG, et al. (1998) Inhibition of IL-12 production by 1,25-dihydroxyvitamin $\mathrm{D}_{3}$. Involvement of NF-kappaB downregulation in transcriptional repression of the p40 gene. J Clin Invest 101, 252-262.

6. Alroy I, Towers TL \& Freedman LP (1995) Transcriptional repression of the interleukin-2 gene by vitamin $\mathrm{D}_{3}$ : direct inhibition of NFATp/AP-1 complex formation by a nuclear hormone receptor. Mol Cell Biol 15, 5789-5799.

7. Menendez C, Lage M, Peino R, et al. (2001) Retinoic acid and vitamin $\mathrm{D}(3)$ powerfully inhibit in vitro leptin secretion by human adipose tissue. J Endocrinol 170, 425-431.

8. Sun X \& Zemel MB (2007) Calcium and 1,25-dihydroxyvitamin $\mathrm{D}_{3}$ regulation of adipokine expression. Obesity (Silver Spring) 15, 340-348

9. Bellia A, Garcovich C, D'Adamo M, et al. (2013) Serum 25-hydroxyvitamin D levels are inversely associated with systemic inflammation in severe obese subjects. Intern Emerg Med 8, 33-40.

10. van de Luijtgaarden KM, Voute MT, Hoeks SE, et al. (2012) Vitamin D deficiency may be an independent risk factor for arterial disease. Eur J Vasc Endovasc Surg 44, 301-306.

11. Vilarrasa N, Vendrell J, Maravall J, et al. (2010) Is plasma 25 $(\mathrm{OH}) \mathrm{D}$ related to adipokines, inflammatory cytokines and insulin resistance in both a healthy and morbidly obese population? Endocrine 38, 235-242.

12. Kim M, Na W \& Sohn C (2013) Correlation between vitamin D and cardiovascular disease predictors in overweight and obese Koreans. J Clin Biochem Nutr 52, 167-171.

13. Vaidya A, Williams JS \& Forman JP (2012) The independent association between 25-hydroxyvitamin D and adiponectin and its relation with BMI in two large cohorts: the NHS and the HPFS. Obesity (Silver Spring) 20, 186-191.
14. Husemoen LL, Skaaby T, Martinussen T, et al. (2014) Investigating the causal effect of vitamin $\mathrm{D}$ on serum adiponectin using a Mendelian randomization approach. Eur J Clin Nutr 68, 189-195.

15. Khoo AL, Chai LY, Koenen HJ, et al. (2011) Regulation of cytokine responses by seasonality of vitamin $\mathrm{D}$ status in healthy individuals. Clin Exp Immunol 164, 72-79.

16. Barker T, Rogers VE, Levy M, et al. (2015) Supplemental vitamin $\mathrm{D}$ increases serum cytokines in those with initially low 25-hydroxyvitamin D: a randomized, double blind, placebocontrolled study. Cytokine 71, 132-138.

17. Asemi Z, Samimi M, Tabassi Z, et al. (2013) Vitamin D supplementation affects serum high-sensitivity C-reactive protein, insulin resistance, and biomarkers of oxidative stress in pregnant women. J Nutr 143, 1432-1438.

18. Witham MD, Dove FJ, Khan F, et al. (2013) Effects of vitamin D supplementation on markers of vascular function after myocardial infarction - a randomised controlled trial. Int J Cardiol 167, 745-749.

19. Abou-Raya A, Abou-Raya S \& Helmii M (2013) The effect of vitamin D supplementation on inflammatory and hemostatic markers and disease activity in patients with systemic lupus erythematosus: a randomized placebo-controlled trial. J Rheumatol 40, 265-272.

20. Grossmann RE, Zughaier SM, Liu S, et al. (2012) Impact of vitamin D supplementation on markers of inflammation in adults with cystic fibrosis hospitalized for a pulmonary exacerbation. Eur J Clin Nutr 66, 1072-1074.

21. Schleithoff SS, Zittermann A, Tenderich G, et al. (2006) Vitamin D supplementation improves cytokine profiles in patients with congestive heart failure: a double-blind, randomized, placebo-controlled trial. Am J Clin Nutr 83, 754-759.

22. Neyestani TR, Nikooyeh B, Alavi-Majd H, et al. (2012) Improvement of vitamin D status via daily intake of fortified yogurt drink either with or without extra calcium ameliorates systemic inflammatory biomarkers, including adipokines, in the subjects with type 2 diabetes. J Clin Endocrinol Metab 97, 2005-2011.

23. Barnes MS, Horigan G, Cashman KD, et al. (2011) Maintenance of wintertime vitamin D status with cholecalciferol supplementation is not associated with alterations in serum cytokine concentrations among apparently healthy younger or older adults. J Nutr 141, 476-481.

24. Pittas AG, Harris SS, Stark PC, et al. (2007) The effects of calcium and vitamin D supplementation on blood glucose and markers of inflammation in nondiabetic adults. Diabetes Care 30, 980-986.

25. Wood AD, Secombes KR, Thies F, et al. (2012) Vitamin $\mathrm{D}_{3}$ supplementation has no effect on conventional cardiovascular risk factors: a parallel-group, double-blind, placebocontrolled RCT. J Clin Endocrinol Metab 97, 3557-3568.

26. Yusupov E, Li-Ng M, Pollack S, et al. (2010) Vitamin D and serum cytokines in a randomized clinical trial. Int J Endocrinol 2010, 305054 .

27. Jorde R, Sneve M, Torjesen PA, et al. (2010) No effect of supplementation with cholecalciferol on cytokines and markers of inflammation in overweight and obese subjects. Cytokine 50, 175-180.

28. Wamberg L, Kampmann U, Stodkilde-Jorgensen $\mathrm{H}$, et al. (2013) Effects of vitamin D supplementation on body fat accumulation, inflammation, and metabolic risk factors in obese adults with low vitamin D levels - results from a randomized trial. Eur J Intern Med 24, 644-649.

29. Carrillo AE, Flynn MG, Pinkston C, et al. (2012) Vitamin D supplementation during exercise training does not alter 
inflammatory biomarkers in overweight and obese subjects. Eur J Appl Physiol 112, 3045-3052.

30. Jorde R, Strand Hutchinson M, Kjaergaard M, et al. (2013) Supplementation with high doses of vitamin D to subjects without vitamin $\mathrm{D}$ deficiency may have negative effects: pooled data from four intervention trials in Tromso. ISRN Endocrinol 2013, 348705.

31. Medzhitov R (2008) Origin and physiological roles of inflammation. Nature 454, 428-435.

32. Trinchieri G (2012) Cancer and inflammation: an old intuition with rapidly evolving new concepts. Annu Rev Immunol 30 677-706.

33. Tran B, Armstrong BK, Carlin JB, et al. (2012) Recruitment and results of a pilot trial of vitamin D supplementation in the general population of Australia. J Clin Endocrinol Metab 97, $4473-4480$

34. Satoh N, Naruse M, Usui T, et al. (2004) Leptin-to-adiponectin ratio as a potential atherogenic index in obese type 2 diabetic patients. Diabetes Care 27, 2488-2490.

35. Institute of Medicine (2011) Dietary Reference Intakes for Calcium and Vitamin D. Washington, DC: The National Academies Press.

36. Hoenig JM \& Heisey DM (2001) The abuse of power: the pervasive fallacy of power calculations for data analysis. Am Stat 55, 19-24.

37. Tilg H, Dinarello CA \& Mier JW (1997) IL-6 and APPs: anti-inflammatory and immunosuppressive mediators. Immunol Today 18, 428-432.

38. Steensberg A, Fischer CP, Keller C, et al. (2003) IL-6 enhances plasma IL-1ra, IL-10, and cortisol in humans. Am J Physiol Endocrinol Metab 285, E433-E437.

39. Willheim M, Thien R, Schrattbauer K, et al. (1999) Regulatory effects of 1alpha,25-dihydroxyvitamin $\mathrm{D}_{3}$ on the cytokine production of human peripheral blood lymphocytes. J Clin Endocrinol Metab 84, 3739-3744.

40. Gepner AD, Ramamurthy R, Krueger DC, et al. (2012) A prospective randomized controlled trial of the effects of vitamin D supplementation on cardiovascular disease risk. PLOS ONE 7, e36617.

41. Beilfuss J, Berg V, Sneve M, et al. (2012) Effects of a 1-year supplementation with cholecalciferol on interleukin-6, tumor necrosis factor-alpha and insulin resistance in overweight and obese subjects. Cytokine $\mathbf{6 0}, 870-874$

42. Breslavsky A, Frand J, Matas Z, et al. (2013) Effect of high doses of vitamin D on arterial properties, adiponectin, leptin and glucose homeostasis in type 2 diabetic patients. Clin Nutr 32, 970-975.

43. Zhang Y, Leung DY, Richers BN, et al. (2012) Vitamin D inhibits monocyte/macrophage proinflammatory cytokine production by targeting MAPK phosphatase-1. I Immunol 188, 2127-2135.

44. Barker T, Martins TB, Hill HR, et al. (2013) Circulating pro-inflammatory cytokines are elevated and peak power output correlates with 25 -hydroxyvitamin $\mathrm{D}$ in vitamin $\mathrm{D}$ insufficient adults. Eur J Appl Physiol 113, 1523-1534.

45. Tran B, Armstrong BK, Ebeling PR, et al. (2014) Effect of vitamin D supplementation on antibiotic use: a randomized controlled trial. Am J Clin Nutr 99, 156-161.

46. Bergman P, Lindh AU, Bjorkhem-Bergman L, et al. (2013) Vitamin D and respiratory tract infections: a systematic review and meta-analysis of randomized controlled trials. PLOS ONE 8, e65835.

47. Chel V, Wijnhoven HA, Smit JH, et al. (2008) Efficacy of different doses and time intervals of oral vitamin D supplementation with or without calcium in elderly nursing home residents. Osteoporos Int 19, 663-671.

48. Hollis BW \& Wagner CL (2013) Clinical review: the role of the parent compound vitamin $\mathrm{D}$ with respect to metabolism and function: why clinical dose intervals can affect clinical outcomes. J Clin Endocrinol Metab 98, 4619-4628.

49. Ish-Shalom S, Segal E, Salganik T, et al. (2008) Comparison of daily, weekly, and monthly vitamin $\mathrm{D}_{3}$ in ethanol dosing protocols for two months in elderly hip fracture patients. J Clin Endocrinol Metab 93, 3430-3435.

50. Binkley N, Gemar D, Engelke J, et al. (2011) Evaluation of ergocalciferol or cholecalciferol dosing, 1,600 IU daily or 50,000 IU monthly in older adults. J Clin Endocrinol Metab 96, 981-988.

51. Vieth R (2008) Comment on Chel et al.: efficacy of different doses and time intervals of oral vitamin D supplementation with or without calcium in elderly nursing home residents. Osteoporos Int 19, 721-722 (author reply 723). 